\title{
ONCOCERCOSE EQÜINA: DIAGNÓSTICO E VERIFICAÇÃO DA HEMATOFAGIA POR SIMULÍDEOS E CULICÓIDES, PROVÁVEIS VETORES NO RIO DE JANEIRO, BRASIL
}

\author{
Regina Helena dos Santos Calvão-Brito ${ }^{1}$ \\ Maria de Lurdes de Azevedo Rodrigues ${ }^{2}$ \\ Elázaro Moses Mokrabe ${ }^{1}$ \\ Marilza Maia-Herzog ${ }^{1}$ \\ Rubens Pinto de Mello ${ }^{1}$ \\ Virgínio Pereira da Silva-Júnior ${ }^{2}$
}

\begin{abstract}
EQUINE ONCHOCERCIASIS: DIAGNOSTIC AND VERIFICATION OF THE HAEMATOPHAGOUS BLACK FLIES AND BITING MIDGES CONSIDERED AS VECTORS IN RIO DE JANEIRO, BRAzIL. Onchocerca cervicalis Railliet \& Henry, 1910 is a nematode that parasite equines. Its ocurrence has already been reported in the States of Mato Grosso and Rio de Janeiro, Brazil. Different diagnostic techniques were used in this study to establish the prevalence of the parasite in Rio de Janeiro State and the biting behaviour of the simuliid and ceratopogonid species suspected of being vectors was studied. All of the biopsies made in the region of the withers, shoulder and umbilicus of 57 animals were negative. Necropsies carried out on 28 equines revealed 4 to be positive for the parasite; 2 for microfilariae and 2 for adults. Simuliid flies showed preference for biting the ventral region and ceratopogonids the lower parts of the legs of horses. It was concluded that a more detailed investigation is required to determine a better diagnostic technique in order to verify prevalence rates of the parasite in horses in the State.

KEY WORDS. Onchocerca cervicalis, equines, black flies, biting-midges, Rio de Janeiro, Brazil
\end{abstract}

Onchocerca cervicalis Railliet \& Henry, 1910 é um nematóide, da família Onchocercidae, sub-família Onchocercinae, que parasita eqüídeos nas Américas do Norte e do Sul, Ásia e Austrália (BAIN 1981). As formas jovens denominadas de microfilárias são encontradas no tecido subcutâneo dos eqüídeos, enquanto as formas adultas localizam-se no ligamento da nuca (MELLOR 1973).

Uma maior concentração de microfilárias ocorre na região anterior à cernelha, na parte posterior às patas dianteiras e no abdome, próximo ao umbigo (CUMMINGS \& JAMES 1985; RABAlais \& VotaVA 1974).

Na Europa e nos Estados Unidos da América, espécies do gênero Culicoides foram incriminadas como vetores deste filarídeo (STEWARD 1935, 1937; MoIGNOUX 1951, 1952). MELLOR (1974) sugere que os simulídeos e os culicóides

1) Departamento de Entomologia, Instituto Oswaldo Cruz. Avenida Brasil 4365, 21045-900 Rio de Janeiro, Rio de Janeiro, Brasil.

2) Departamento de Parasitologia Animal, Universidade Federal Rural do Rio de Janeiro. BR 465 Km 47, 23851-970 Seropédica, Rio de Janeiro, Brasil.

Revta bras. Zool. 15 (3): 583 - 587, 1998 
poderiam ser vetores de $O$. cervicalis, pois se alimentam superficialmente, atingindo somente até os capilares, estando bem adaptados à ingestão de microfilárias.

O local de preferência dos culicóides para realizar a hematofagia é a parte inferior das patas (TOWNLEY et al. 1984).

No Brasil, a ocorrência de O. cervicalis foi relatada por BRAZ (1971) e RODRIGUES et al. (1996). RODRIGUES et al. (1996) verificaram uma prevalência de $75 \%$ de $O$. cervicalis em cavalos da região de Itaguaí. O presente trabalho tem como objetivos verificar a prevalência da oncocercose eqüina utilizando diferentes técnicas de diagnóstico e estudar a hematofagia, segundo locais de preferência no corpo do eqüino, dos prováveis vetores de $O$. cervicalis no estado do Rio de Janeiro.

\section{MATERIAL E MÉTODOS}

Três técnicas foram utilizadas para verificar a presença de $O$. cervicalis no eqüino: pesquisa de microfilárias dérmicas através de biópsia, amostragem de tecido subcutâneo durante necrópsia e pesquisa de adultos no ligamento da nuca.

Vinte e oito animais foram necropsiados, dos quais 20 foram biopsiados, sete dias antes do sacrifício. Em outros 57 eqüinos, foram feitas apenas biópsias. Os animais examinados eram provenientes dos Municípios de Araruama (11 animais), Nilópolis (12 animais), Itaguaí (26 animais) e Rio de Janeiro (8 animais) na faixa etária entre 2 e 25 anos. Os animais necropsiados foram doados à Universidade Federal Rural do Rio de Janeiro, pelo Serviço de Apreensão do Departamento Nacional de Estradas e Rodagem (DNER).

As biópsias e amostragem de tecido subcutâneo coletado durante a necrópsia foram realizadas nas regiões da cernelha, da espádua e umbilical. Na biópsia foi retirado um fragmento de tecido subcutâneo de aproximadamente $2 \mathrm{~cm}$ de diâmetro e na necrópsia, o mesmo foi de $15 \mathrm{~cm}$. A técnica utilizada para estimular a saída das microfilárias do tecido foi preconizada por BEVILAQUA et al. (1993a).

Um eqüino foi utilizado como isca para coleta de simulídeos e culicóides, no período de maio a dezembro de 1995, visando verificar as áreas preferenciais para hematofagia dos prováveis vetores de $O$. cervicalis. Os insetos foram coletados sobre o eqüino utilizando o capturador de Castro (MAIA-HERzoG et al. 1988) e dissecados para pesquisa de larvas de $O$. cervicalis. Os resultados foram anotados em planilha delimitando as áreas corporais do animal conforme mapeamento descrito por TOWNLEY et al. (1984).

Dos insetos capturados $10 \%$ dos 3593 simulídeos e $10 \%$ dos 74 culicóides, foram utilizados para pesquisa de formas larvares de $O$. cervicalis, através da técnica com coloração por hematoxilina, visando comprovar os possíveis vetores.

\section{RESULTADOS E DISCUSSÃO}

Todos os animais examinados pela técnica de biópsia apresentaram-se negativos. Dois animais estavam positivos ao serem examinados para a pesquisa de microfilárias dérmicas, através da amostragem de tecido subcutâneo durante a necrópsia. Dois cavalos foram positivos à pesquisa de adultos no ligamento da nuca. 
Um dos animais positivos para microfilárias dérmicas não apresentou adultos de $O$. cervicalis no ligamento da nuca (Tab. I).

Tabela I. Técnicas utilizadas para exame de eqüídeos verificando a ocorrência de Onchocerca cervicalis.

\begin{tabular}{|c|c|c|c|c|c|c|}
\hline \multirow{2}{*}{ Procedência } & \multicolumn{6}{|c|}{ Técnicas empregadas } \\
\hline & \multicolumn{2}{|c|}{ Biópsia } & \multicolumn{2}{|c|}{ Amostragem } & \multicolumn{2}{|c|}{ Pesquisa de adultos } \\
\hline Araruama & 11 & 0 & 11 & 0 & 11 & 0 \\
\hline Nilópolis & 12 & 0 & - & - & - & - \\
\hline Itaguaí (DNER/UFRRJ) & 26 & 0 & 17 & 2 & 17 & 2 \\
\hline Rio de Janeiro & 8 & 0 & - & - & - & - \\
\hline Total & 57 & 0 & 28 & 2 & 28 & 2 \\
\hline
\end{tabular}

A elevada prevalência encontrada por RoDRIGUES et al. (1996) para o Município de Itaguaí, não foi observada para Araruama sugerindo que neste talvez não ocorra o filarídeo ou que a amostra estudada tenha que ser aumentada, visando confirmar os resultados observados até o momento. Este fato e a idade dos animais são as possíveis explicações para a baixa prevalência observada.

A principal diferença entre as técnicas de diagnóstico para a pesquisa de microfilárias dérmicas foi o tamanho da amostra de pele utilizada, o que provavelmente, pode influenciar os melhores resultados obtidos através da técnica de amostragem de pele durante a necrópsia. Outro fator que pode ter influenciado os baixos valores obtidos seriam as áreas de concentração de microfilárias. Estudos realizados em países de clima temperado indicam a cernelha e a região umbilical como sendo os principais locais de concentração de microfilárias. No entanto, em países de clima tropical, ainda não foi realizado qualquer estudo sobre a distribuição e zonas de concentração das microfilárias, no corpo do eqüino. Vários trabalhos realizados com bovinos demonstraram que existem microfilárias de $O$. gutturosa, distribuídas por todo o corpo do animal, porém existem áreas de maior concentração e as amostras devem ser coletadas nestes locais BEVILAQUA et al. (1993b).

Os simulídeos buscavam com maior frequêencia a região ventral, próximo à genitália, sendo o local de preferência, com maior percentual. Os culicóides buscavam a parte inferior das patas, especialmente a quartela. Através da figura 1, pode-se observar o mapeamento das áreas preferencias para a alimentação. MELLOR (1974), afirma que os culicóides são os vetores de $O$. cervicalis e sugere que os simulídeos também seriam vetores em potencial devido à característica do hematofagismo superficial, atingindo somente até os capilares, porém até o momento, a atuação dos simulídeos como vetor de $O$. cervicalis não foi comprovada. Larvas infectantes $\left(\mathrm{L}_{3}\right)$ de $O$. linealis desenvolveram-se em Simulium ornatum em infecções experimentais (DoHnal et al. 1990).

Os simulídeos e os culicóides encontrados exercendo a hematofagia, não estavam positivos para microfilárias, porém, devemos considerar a baixa parasitemia dos eqüídeos examinados. 


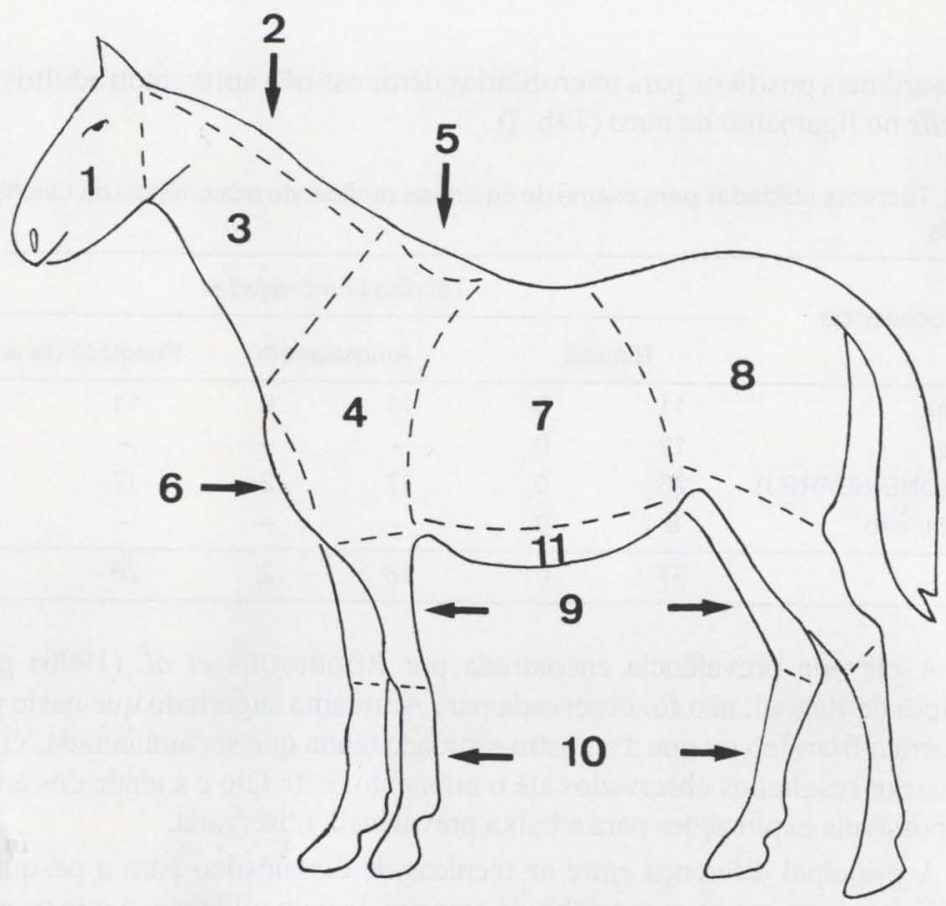

Fig. 01. Mapeamento das áreas preferenciais para alimentaçăo dos vetores hematófagos. (1) Cabeça, (2) crina, (3) pescoço, (4) espádua, (5) cernelha, (6) peito, (7) lateral, (8) quarto traseiro, (9) parte superior das patas, (10) parte inferior das patas, (11) abdômen.

AGRADECIMENTOS. Aos responsáveis pelos eqüídeos da Defesa Civil de Queimados, da UFRRJ, do Centro de Controle de Zoonoses e do Campo de Instrução do Gericinó, em especial ao Médico-Veterinário Dr. Paulo Varella, pela autorização e realização das biópsias. Aos Médicos-Veterinários Marco Antonio e Márcia Medeiros de Araújo, alunos do Curso de Pós-Graduação da Universidade Federal Rural do Rio de Janeiro pela realização de parte das biópsias. Ao Dr. Jürgen Doberainer, pesquisador da EMBRAPA pelo apoio logístico durante a excução deste trabalho. As estagiárias Débora Henrique dos Santos Anjos, do Instituto de Biologia, Universidade Federal do Rio de Janeiro e Ana Margarida Ribeiro do Amaral, do Setor de Simulídeos, do Instituto Oswaldo Cruz, pelo apoio durante a realização do trabalho de campo. Ao Conselho Nacional de Desenvolvimento Científico e Tecnológico $(\mathrm{CNPq})$ pelo auxílio concedido para a execução deste trabalho.

\section{REFERÊNCIAS BIBLIOGRÁFICAS}

BAIN, O. 1981. Le gene Onchocerca: hypotheses sur on evolution et dé dichtomique des espéces. Ann. Parasitol. Hum. Comp. 56: 503-526.

BEVILAQUA, C.M.; L.Y.ACHI \& P. DoRCHIES. 1993a. Distribution des microfilaires d'Onchocerca spp. dans la peau de bovinas dans le Sud Ouest de la France. Rev. Méd. Vét. 144 (1): 57-59. 
1993b. Étude de la prévalence de l'Onchocercose bovine dans le Sud-Ouest de la France. Rev. Méd. Vét. 144 (2): 56-58.

BRAZ, F.F. 1971. Onchocerca cervicalis em Eqüínos. Arqs Biol. Tecnol. 14 (1): $1-42$.

Cummings, E. \& E.R. James. 1985. Prevalence of equine onchocerciasis in southeastern and midwestern United States. Jour. Amer. Vet. Med. Ass. 186 (11): 1202-1203.

Dohnal, J.; J. BLINN; G. WAHL \& H. SCHULZ-KeY. 1990. Distribution of microfilariae of Onchocerca linealis and Onchocerca gutturosa in the skin of cattle in Germany and their development in Simulium ornatum and Culicoides nubeculosus following artificial infestation. Vet. Parasitol. 36: 325-332.

Maia-Herzog, M.; M.L. Felipe-Bauer \& R. Malaguti. 1988. A contribution to the study of Simulium and Culicoides of Rio de Janeiro: monthly incidence and biting activity. Mems. Inst. Oswaldo Cruz, Rio de Janeiro, 83 (1): 95-99.

MELloR, P.S. 1973. Studies on Onchocerca cervicallis Railliet and Henry, 1910. I. Onchocerca cervicallis in British horses. Jour. Helminth. 48: 145-153. 1974. Studies on Onchocerca cervicallis Railliet and Henry, 1910: V. The development of Onchocerca cervicallis larvae in the vectors Culicoides nubeculosus, C. variipennis. Jour. Helm. 49 (1): 33-42.

MoIGNouX, J.B. 1951. Recherches experimentales sur le cycle evolutif des microfilares Onchocerca reticulata Diesing, en Camargue. Comples rendus des seances de la Societe de biologie 145: 1572.

1952. Sur l'identite des deux especes d' Onchocerca O. reticulata Diesing 1841 et $O$. cervicallis Rail. and Henry 1910. Estudes biologique comparative. Comples rendus des seances de la Societe de Biologie 146: 573.

RABALAIS, F.C. \& C.L. VOTAVA. 1974. Cutaneous distribution of microfilariae of Onchocerca cervicalis. Amer. Jour. Vet. Res. 35 (10): 1369-1370.

Rodrigues, L.; D. Anjos; M. Oliveira \& M. Souto-Maior. 1996. Prévalence d'Onchocerca cervicalis chez des equidés dans l'état de Rio de Janeiro, Brésil. Rev. Méd. vét. 147 (10): 1-2

STEWARD, J.S. 1935. Fistulous withers and poll evil. Vet. Rec. 15: 1563.

1937. The ocurrence of Onchocerca gutturosa Neumann in cattle in England with an account of its life - history and development in Simulium ornatum Meigen. Parasitology 29: 21.

TOWNLEY, P.; K.P. BAKER \& P.J. QUINN. 1984. Preferential landing and engorgin sites of Culicoides species landing on a horse in Ireland. Eq. Vet. Jour. 16 (2): 117-120.

Recebido em 30.I.1997; aceito em 25.VI.1998. 